\title{
MOLECULAR DIAGNOSIS OF LUNG CANCER
}

\section{Savelina L. Popovska, Tereza B. Dineva ${ }^{1}$, Polina D. Damyanova ${ }^{1}$}

Department of Pathology, Medical University - Pleven, Bulgaria

${ }^{1}$ Section of Molecular Pathology, University Scientific Research Laboratory, Medical University - Pleven, Bulgaria

\section{Corresponding Author:}

Savelina L. Popovska

Department of Pathology,

Medical University - Pleven

1, St. Kl. Ohridski Str.

Pleven, 5800

Bulgaria

e-mail: sapopovska@yahoo.com

Received: January 16, 2018

Revision received: January 30, 2018

Accepted: February 27, 2018

\section{Summary}

Lung cancer is still the leading cancer-related cause of death with a high incidence rate in the whole world. Treatment options - surgery, radiotherapy, and chemotherapy, depending on the stage of the disease. During the last decade, many molecular alterations were discovered that led to impressive changes in treatment. Personalized approaches, including target therapies with specific inhibitor drugs, became a part of the standard therapies. This article reviews current molecular biomarkers used in clinical practice to treat lung cancer patients.

Key words: molecular biomarkers, lung cancer, targeted therapies

\section{Introduction}

Lung cancer is the leading cause of cancer-related mortality in the whole world. It causes the highest number of deaths, the latter being on the increase [1]. Non-small cell lung cancer (NSCLC), which accounts for about $80 \%$ of all lung cancer, is still a big problem for healthcare systems due to poor response rates, poor prognosis and the insufficient 5-year survival rate of about 14\%. In Bulgaria, based on the annual reports from the National Bulgarian Cancer Registry, lung cancer was reported as the most common malignancy in males in 2012 , representing $19.1 \%$ of all the new cases in men, and the seventh most common in females ( $5.1 \%$ of all the new cases in females). For 2012, 3594 lung cancer deaths were reported, of which male cases were four times the number of the female cases. The expected new lung cancer cases for 2015 were 4081, and expected deaths were 3622 [2]. The role of genetic factors in lung cancer has become more recognizable during the last couple of years. Mutational analysis and genomics profiling have contributed to new developments in the field of lung cancer in Bulgaria and have helped to define the critical role of some mutations in the progression of the disease [3-5]. Testing for genetic alterations has attained great clinical importance in the recent years, and some molecular biomarkers have become reliable targets for specifically targeted drugs in NSCLC. As can be expected, treatment for lung cancer has also changed. 
Furthermore, this has encouraged researchers to seek new biological markers to better explain lung carcinogenesis [6].

This article aims to summarize the biomarkers of importance for the current clinical practice in lung cancer treatment.

\section{Discussion}

Extensive research in the field of lung cancer has provided new scientific clues to better understanding the pathobiology of NSCLC, and the small molecules that target certain genetic alterations were introduced into routine clinical practice. The paradigm for treatment of lung cancer has changed significantly [7-9]. The well-known biological therapies in clinical practice target specific genetic alterations in NSCLC, mutations in RAS and epidermal growth factor receptor (EGFR) oncogenes, the ALK rearrangement or inactivated tumor suppressor genes like TP53 [10]. RAS proteins are responsible for controlling the signaling pathways in the cell, defining growth, survival, and differentiation of the cell itself. Since the activation of RAS proteins is under strict control in the normal cell, a change in the RAS signaling could lead to malignant transformation. RAS genes were first identified in the 20-th century as retroviral oncogenes from the genome rat sarcoma viruses [11]. KRAS mutations are predominately found in adenocarcinomas and are seen in approximately $25 \%$ of cases. Although KRAS has been found as the most frequently mutated gene in NSCLC, there is still no targeted drug available in the clinical practice. Alteration if BRAF, a downstream gene of KRAS signaling, has been found in NSCLC, though with a low frequency that ranges from one to five percent $[12,13]$. The PI3K/AKT/ mTOR pathway is another signaling pathway that is dysregulated in NSCLC and can be easily assessed by next-generation sequencing (NGS).

Some subtypes of NSCLC are characterized by specific mutations in the gene for EGFR, which defines their sensitivity to certain inhibitors of the associated oncogenic signaling pathways. According to the established rule, they are more common in women, nonsmokers, and subjects of Asian origin. EGFR is a known member of the big HER family [14]. EGFR mutations are divided into common (del exon
19, point mutation L858R), and uncommon mutations. Common mutations are considered to be the target for most tyrosine kinase inhibitors (TKIs). For Bulgaria, the prevalence of EGFR mutation frequency in NSCLC is considered equal to the worldwide reported data [15]. So far, the EGFR TKIs first (gefitinib, erlotinib) and second generation (afatinib) routinely used in the clinical practice have proven their efficacy in NSCLC patients harboring activating EGFR mutations by showing a significant change in the survival rates, as compared to standard platinumbased regimens [16, 17].

Table 1. Frequency of mutations in NSCLC by Vanderbilt-Ingram Cancer Center (2016) [17]

\begin{tabular}{lll}
\hline Gene & Alteration & $\begin{array}{l}\text { Frequency in } \\
\text { NSCLC }\end{array}$ \\
\hline AKT1 & mutation & $1 \%$ \\
\hline ALK & rearrangement & $3-7 \%$ \\
\hline BRAF & mutation & $1-3 \%$ \\
\hline DDR2 & mutation & $-4 \%$ \\
\hline EGFR & mutation & $10-35 \%$ \\
\hline FGFR1 & amplification & $20 \%$ \\
\hline HER2 & mutation & $2-4 \%$ \\
\hline KRAS & mutation & $15-25 \%$ \\
\hline MEK1 & mutation & $1 \%$ \\
\hline METa & amplification & $2-4 \%$ \\
\hline NRAS & mutation & $1 \%$ \\
\hline PIK3CA & mutation & $1-3 \%$ \\
\hline PTEN & mutation & $4-8 \%$ \\
\hline RET & rearrangement & $1 \%$ \\
\hline ROS1 a & rearrangement & $1 \%$ \\
\hline & &
\end{tabular}

However, EGFR T790M mutation (also known as a gate-keeper) is the reason for resistance to most clinically available first and second generation EGFR TKIs, which practically explains the progression during treatment with TKIs in about $60 \%$ of the cases, with a median time to progression about ten months. The T790M mutation has to be selectively targeted by a new, third generation of TKIs to overcome the EGFR resistance, such as osimertinib, rociletinib, and others, that are still clinically tested. T790M is only one way to overcome the resistance to EGFR TKIs because this resistance is complex and due to various mechanisms. These mechanisms included activation of alternative 
pathways (like c-Met), changes in downstream pathways (such as K-RAS mutations or loss of PTEN), faults in the EGFR-TKIs-mediated apoptosis pathway, or subsequent change in the histology subtype [18]. Targeted therapies are used in patients with adenocarcinoma of the lung since genetic activating mutations are usually found in the group of patients with this histologic subtype. The mechanisms of acquired resistance to EGFR-TKIs are summarized in Figure 1 [19].

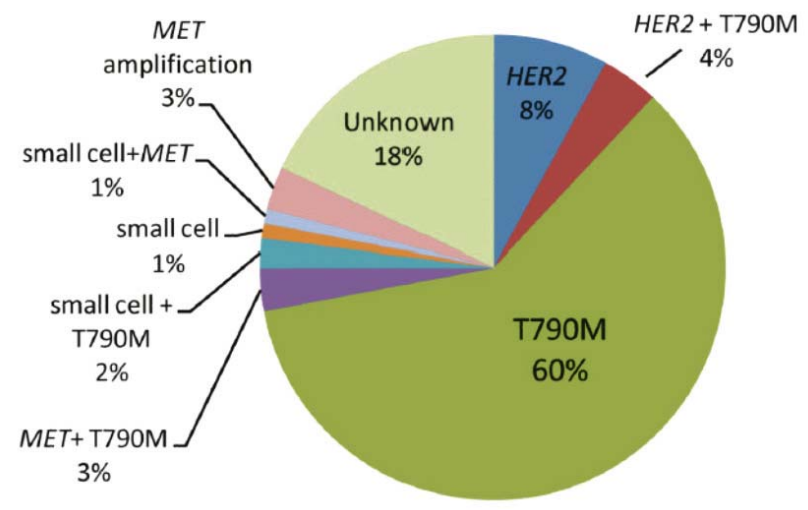

Figure 1. Mechanisms of acquired resistance to EGFR-TKIs by Yu HA et al (2013) [19]

The functions of the cytokine vascular endothelial growth factor (VEGF) include stimulation of the cell growth and angiogenesis by increasing microvascular permeability. Based on the discovery of its functions, the antiVEGF monoclonal antibody bevacizumab was introduced as the first-line therapy in patients with non-squamous NSCLC. This treatment is combined with platinum doublet, followed by a single agent maintenance monotherapy with bevacizumab. Two phase III trials have proven that adding bevacizumab to chemotherapy significantly increased progression-free survival and response rates [20,21]. Since bevacizumab is limited only to the non-squamous histology of the lung and pemetrexed is proven to be most effective in cases of adenocarcinoma, the therapy with pemetrexed/cisplatinum for adenocarcinoma and the platinum doublet with bevacizumab is reported as first-line standard therapy in patients with non-squamous NSCLC in the absence of any activating mutation [22, 23].

All previous statements provide evidence that treatment of lung cancer is moving from standard histology-based therapy to the personalized approach. This evidence is based on the individual's genetic alterations, and the treatment strategy should be defined by the genetic and pharmacogenetic profile. In such a way, resistance is overcome, and acute therapy-related side effects are avoided, thus increasing the chances for a positive treatment outcome. The idea of precision medicine relies on a deep understanding of the cancer genome as determined by NGS.

Currently, technology has made it easier to implement NGS not only in research but also in routine clinical practice in a cost-effective manner. NGS-based diagnostics can provide clinically relevant information from readily available formalin-fixed paraffin-embedded (FFPE) tissue, which is of utmost importance for daily clinical practice. Besides, it could be easily incorporated into standard clinical practice [24]. The current practice of single mutation testing NSCLC patients is going to be duly replaced by multiple gene alterations testing, using NGS technology for more reliable and timely diagnosis.

Also, if a patient has failed standard cytotoxic or targeted therapy, NGS could be of great help for identifying the exact clinical trials into which the patient could be possibly enrolled. The individual approach to a single patient is the main issue of current oncological practice. NGS is the way to face that major challenge by resolving the problems concerning drug efficacy and safety. The individual sensitivity of every single patient is defined, based on the patient's genetic landscape. NGS provides information why some patients experience extreme toxicity to certain drugs, while others remain subdosed by the very same dose schedule. The scientific 
discoveries, which NGS has brought to oncology practice and the updated genetic database in the last couple of years, emphasize the role of pharmacogenomics in individualizing therapy in the era of precision medicine [25-27].

Resistance to drugs is one of the major causes of therapy failure. Response to therapy is crucial for treatment outcome and success of the treatment applied. That is why researchers have been paying much more attention to the search of genes and genetic variants involved in response to therapy. The literature provides sparse information about the genetic polymorphisms that define the individual therapeutic sensitivity in lung cancer patients. Some polymorphisms in already known genes have been reported to modify resistance to platinum-based regimens, thereby defining therapeutic response and survival. The study of Tibaldi et al. (2008) [28] demonstrated the association between XPD Asp(312)Asn, Lys(751)Gln, ERCC1 C118T, CDA Lys(27)Gln polymorphisms and response, toxicity profile, time to progression, and overall survival (OS) in 65 patients with advanced stage NSCLCL on cisplatin-gemcitabine as first-line treatment. Results of that particular trial could not show any association between ERCC1 and XPD polymorphisms to both response and clinical outcome [28].

Toxicity is of huge importance in the treatment for lung cancer since therapy-related side effects are often a reason for therapy interruptions and subsequent insufficient treatment effects $[29,30]$. Gene repair capacity is influenced by single nucleotide polymorphisms (SNPs) which could explain the differences in the response rates to cytotoxic therapy and therapy failure, as well the degree of toxicity. P53, the tumor suppressor gene of extreme importance for the cell cycle, plays the role of regulator of the cellular processes, including DNA repair and the cell death induced by chemotherapy-related cell damages [31]. The roles mentioned above of P53 for the normal function of the cell cycle prove its great significance because inhibiting its activity could lead to chemoresistance. According to recent studies, P53 gene could harbor a lot of SNPs, but SNP [(rs)1042522 (Arg72Pro)] refers to significant changes in P53 cell function [32]. P53 Arg72Pro polymorphism is associated with lung cancer risk and sensitivity to platinum-based chemotherapy [33]. Some of these biomarkers have been shown to improve anticancer treatment efficacy and reduce toxicity, which could subsequently lower the overall health-care costs. Since research in oncology is expanding, the cost of the studies is of extreme importance. The recently used NGS method, as well as those used in Bulgarian studies, suggest costeffective sequencing and easier data analysis, which is important for the rapidly developing field of oncology. No doubt, the future of cancer genomics is associated with high-throughput sequencing technologies.

\section{Conclusions}

A lot has changed in the treatment of lung cancer in the last decade. Introducing the TKIs as a routine tool in clinical practice has led to significant changes in the progression-free survival rates, thus giving lung cancer patients with the advanced and metastatic disease a new reliable therapeutic possibility and opening new treatment horizons for physicians. However, oncologists still face the need of new targeted approaches to cover the increasing challenge of treatment resistance and related side effects, which influence not only clinical outcomes but patient's quality of life as well. Answers to all clinical questions are meant to be found in genetic discoveries, which have changed the diagnostic approach in lung cancer. Although single molecular biomarkers are now a routine part of diagnosing and treatment of patients with advanced NSCLC, some of them already a diagnostic routine in practice; many others are still being investigated. Therefore, there is a great need for incorporating high profile genetic technologies in diagnosing, treatment and prediction of outcome in clinical practice.

\section{Acknowledgements}

This systematic review was not a part of study and was not supported by any funding source.

\section{References}

1. Centers for Disease Control and Prevention. Compressed mortality file 1999-2011 [CDROM]. Atlanta: National Center for Health Statistics; 2014. 
2. National Oncological Hospital, Bulgarian National Cancer Resistry. Cancer Incidence in Bulgaria. Sofia: Paradigma; 2014, 170 p.

3. Chilingirova N, Balabanski L, Toncheva D, Kurteva G, Damyanov D, Chilingirov P. Identifying the genetic landscape of squamous cell carcinoma (SCC) and adenosquamous carcinoma (ASC) of the lung using nextgeneration sequencing (NGS). Ann Oncol. 2015;26 Suppl: i1- i5.

4. Chilingirova N, Balabanski L, Kurteva G, Toncheva D, Damyanov D, Chilingirov P. Detection of driver mutation heterogeneity in patients with non-small cell lung cancer (NSCLC) using next generation sequencing (NGS). EJC. 2015;51 Suppl 3:S612-S613.

5. Chilingirova $\mathrm{N}$, Balabanski $\mathrm{L}$, Ivanov $\mathrm{S}$, Vazharova R, Toncheva D, Kurteva G. Detection of extensive driver mutation heterogeneity in 5 Bulgarian patients with adenosquamous carcinoma of the lung. Eur J Hum Genet. 2015;23(1).

6. Chilingirova N. [Non-small cell lung cancer in the era of precision medicine]. INSPIRO. 2017;2(40):13-7. Bulgarian.

7. Chilingirova N. [Role of tumor markers]. Rheumatology. 2013;21(1). Bulgarian.

8. Chilingirova N, Kurteva G, Toncheva D. [Lung Cancer and genetics]. Oncology. 2015; 4:40. Bulgarian.

9. Chilingirova N. [Lung Cancer - an overview]. Zdraven Navigator. 2017;3:2-4. Bulgarian.

10. Rousseau B, Jacquot, C, Le Palabe J, Malleter $\mathrm{M}$, Tomasoni C, Boutard T, et al. TP53 transcription factor for the NEDD9/HEF1/ Cas-L gene: potential targets in Non-Small Cell Lung Cancer treatment. Sci Rep. 2015 May 26;5:10356.

11. Malumbres M, Barbacid M. RAS oncogenes: the first 30 years. Nat Rev Cancer. 2003;3(6):45965.

12. Cardarella S, Ogino A, Nishino M, Butaney $\mathrm{M}$, Shen J, Lydon C et al. Clinical, pathologic and biologic features associated with BRAF mutations in non-small cell lung cancer. Clin Cancer Res. 2013;19(16):4532-40.

13. Marchetti A, Felicioni L, Malatesta S, Grazia Sciarrotta M, Guetti L, Chella A, et al. Clinical features and outcome of patients with non-smallcell lung cancer harboring BRAF mutations. J Clin Oncol. 2011;29(26):3574-9.

14. Hynes NE, Lane HA. ERBB receptors and cancer: the complexity of targeted inhibitors. Nat Rev Cancer. 2005;5(5):341-4.

15. Popovska S, Chilingirova N, Dineva $T$, Kamburova Z. Prevalence of epidermal growth factor receptor (EGFR) gene mutations in non-small cell lung cancer (NSCLC) in Bulgarian population, $\mathrm{J}$ Thorac Oncol. 2016;11(4);Suppl:S128-S129.

16. Lampaki S, Lazaridis G, Zarogoulidis K, Kioumis I, Papaiwannou A, Tsirgogianni K, et al. Defining the role of tyrosine kinase inhibitors in early stage non-small cell lung cancer. J Cancer. 2015;6(6):568-74.

17. Vanderbilt-Ingram Cancer Center. Molecular Profiling of Lung Cancer [Internet]. Nashville; c2016. [cited 2018 Febr 18]. Available from: https://www.mycancergenome.org/content/ disease/lung-cancer/.

18. Huang L, Fu L. Mechanisms of resistance to EGFR tyrosine kinase inhibitors. Acta Pharm Sin B. 2015;5(5):390-401.

19. Yu HA, Arcila ME, Rekhtman N, Sima CS, Zakowski MF, Pao W, et al. Analysis of tumor specimens at the time of acquired resistance to EGFR-TKI therapy in 155 patients with EGFR-mutant lung cancers. Clin Cancer Res. 2013;19(8):2240-7.

20. Sandler A, Gray R, Perry MC, Brahmer J, Schiller JH, Dowlati A, et al. PaclitaxelCarboplatin Alone or with Bevacizumab for Non-Small-Cell Lung Cancer. N Engl J Med. 2006;355(24):2542-50.

21. Reck M, von Pawel J, Zatloukal P, Ramlau R, Gorbounova V, Hirsh V, et al. Overall survival with cisplatin-gemcitabine and bevacizumab or placebo as first-line therapy for nonsquamous non-small-cell lung cancer: results from a randomised phase III trial (AVAiL). Ann Oncol. 2010;21(9):1804-9.

22. Reck M, von Pawel J, Zatloukal P, Ramlau R, Gorbounova V, Hirsh V, et al. Phase III trial of cisplatin plus gemcitabine with either placebo or bevacizumab as first-line therapy for nonsquamous non-small-cell lung cancer: AVAil. J Clin Oncol. 2009;27(8):1227-34.

23. Scagliotti G, Hanna N, Fossella F, Sugarman $\mathrm{K}$, Blatter J, Peterson P, et al. The differential efficacy of pemetrexed according to NSCLC histology: a review of two Phase III studies. Oncologist. 2009;14(3):253-63.

24. Chilingirova N, Hammoudeh Z, Balabanski L, Ivanov $\mathrm{S}$, Vazharova $\mathrm{R}$, Nikolova $\mathrm{D}$, et al. TruSight Cancer Sequencing Panel reveals pharmacogenetic variants associated with sensitivity to chemotherapy in lung cancer. memo-Magazine of European Medical Oncology. 2016; 9(1):30-8.

25. Chilingirova N, Kurteva G, Toncheva D. TruSight Cancer Sequencing Panel for detecting of driver mutation heterogeneity and pharmacogenetic variants associated with sensitivity to chemotherapy in patients with non- 
small cell lung cancer (NSCLC). Proceedings of the 8th National Conference for Rare Diseases and Orphan Drugs \& 12th Balkan Congress of Human Genetics; 2017 Sept 8-10; Plovdiv, Bulgaria: Abstract book, p.67.

26. Chilingirova N, Hammoudeh Z, Balabanski L, Ivanov S, Vazharova R, Nesheva D, et al. Pharmacogenomic study of non-small cell lung cancer. Eur J Hum Genet. 2015;23(1).

27. Chilingirova N, Chilingirov N. [Pharmacogenetics and drug resistance in oncology]. Rheumatology. 2017 Dec: 35. Bulgarian.

28. Tibaldi C, Giovannetti E, Vasile E, Mey V, Laan AC, Nannizzi S, et al. Correlation of CDA, ERCC1, and XPD polymorphisms with response and survival in gemcitabine/ cisplatintreated advanced non-small cell lung cancer patients. Clin Cancer Res. 2008;14(6):1797-3.
29. Chilingirova N, Chilingirov N. [Therapy related side effects in the treatment of lung cancer]. Rheumatology. 2017 Dec; 1:47. Bulgarian.

30. Chilingirova N. [Mucositis]. In: [Kurteva G, Trifonova I, editors. Treatment related side effects]. Sofia; 2017. p. 149-163. Bulgarian.

31. Hrstka R, Coates PJ, Vojtesek B. Polymorphisms in p53 and the p53 pathway: roles in cancer susceptibility and response to treatment. J Cell Mol Med. 2009;13(3):440-53.

32. Dumont P, Leu JI, Della Pietra AC 3rd, George DL, Murphy M. The codon 72 polymorphic variants of p53 have markedly different apoptotic potential. Nat Genet. 2003;33(3):35765.

33. Liu L, Wu C, Wang $\mathrm{Y}$, Zhong $\mathrm{R}$, Duan $\mathrm{S}$, Wei $\mathrm{S}$, et al. Combined effect of genetic polymorphisms in P53, P73, and MDM2 on non-small cell lung cancer survival. J Thorac Oncol. 2011;6(11):1793-800. 